\title{
Implementation and Success Factors from Thailand's 1-3-7 Surveillance Strategy for Malaria Elimination
}

\author{
Cheewanan Lertpiriyasuwat \\ DVBD \\ Prayuth Sudathip \\ DVBD \\ Suravadee Kitchakarn \\ DVBD \\ Darin Areechokchai \\ DVBD \\ Sathapana Naowarat \\ RTI International \\ Jui A. Shah ( $\square$ juishah@rti.org ) \\ RTI International https://orcid.org/0000-0001-5723-4738 \\ David Sintasath \\ USAID \\ Niparueradee Pinyajeerapat \\ USAID \\ Felicity Young \\ RTI International \\ Krongthong Thimasarn \\ $\mathrm{n} / \mathrm{a}$ \\ Deyer Gopinath \\ WHO: Organisation mondiale de la Sante \\ Preecha Prempree \\ DVBD
}

Case study

Keywords: Elimination, surveillance, 1-3-7 strategy

Posted Date: March 13th, 2021

DOI: https://doi.org/10.21203/rs.3.rs-295766/v1

License: @ (1) This work is licensed under a Creative Commons Attribution 4.0 International License. Read Full License 


\section{Abstract}

Thailand's National Malaria Elimination Strategy 2017-2026 introduced the 1-3-7 strategy as a robust surveillance and response approach for elimination that would prioritize timely, evidence-based action. Under this strategy, cases are reported within 1 day, cases are investigated within 3 days, and foci are investigated and responded to within 7 days, building on Thailand's long history of conducting case investigation since the 1980s. However, the hallmark of the 1-3-7 strategy is timeliness, with strict deadlines for reporting and response to accelerate elimination. This paper outlines Thailand's experience adapting and implementing the 1-3-7 strategy, including success factors such as a cross-sectoral Steering Committee, participation in a collaborative regional partnership, and flexible local budgets. The program continues to evolve to ensure prompt and high-quality case management, capacity maintenance, and adequate supply of lifesaving commodities based on surveillance data. Results from implementation suggest the 1-3-7 strategy has contributed to Thailand's decline in malaria burden; this experience may be useful for other countries aiming to eliminate malaria.

\section{Background}

In line with the World Health Organization's (WHO's) Global Technical Strategy for Malaria 2016-2030 (GTS) and the subsequent Framework for Malaria Elimination and Malaria Surveillance, Monitoring \& Evaluation reference manual [1], the countries in the Greater Mekong Subregion (GMS) have endorsed the goals of eliminating Plasmodium falciparum by 2025 and all forms of malaria by 2030 [1]. The GMS comprises Cambodia, Lao People's Democratic Republic, Myanmar, Vietnam, and Thailand, which are all showing notable declines in malaria burden [2]. GMS countries are approaching the challenge using several tailored malaria elimination strategies, including distribution of insecticide-treated bed nets (ITNs), expanded networks of village health workers, and case-based surveillance and response $[3,4]$.

Thailand's National Malaria Elimination Strategy 2017-2026 (NMES), which outlines a vision for reaching zero indigenous cases by 2024, was approved by the Cabinet of the Royal Thai government in 2016. A complementary Operational Plan 2017-2021 proposed a national budget of 2.3 billion Thai baht (USD $\$ 64.8$ million) over the next 5 years to support malaria elimination strategies to interrupt transmission [5]. The core focus of these plans is to identify infections rapidly and to use timely and active surveillance and response to prevent them from spreading [5]. The NMES introduced the 1-3-7 strategy as a robust surveillance and response approach for elimination that would prioritize timely, evidence-based action. The strategy requires notification of each malaria case within 1 day of diagnosis, investigation and classification of each case within 3 days, and a focus investigation and response within 7 days to deploy needed interventions.

The 1-3-7 strategy builds on Thailand's history since the 1980s of conducting case investigation and classification. The Containment Project, which was implemented from 2008 to 2012, served as precursor to real-time case-based data collection and follow-up and led to the launch of electronic data capture in a national web-based malaria information system (MIS) known as "Malaria Online [6]." In 2009, Thailand added foci investigation as a central part of its malaria control strategy [7]. With the launch of the NMES in 2017, Thailand's Division of Vector Borne Diseases (DVBD) within the Department of Disease Control (DDC) of the Ministry of Public Health (MOPH) upgraded Malaria Online to aggregate all sources of malaria case data and monitor progress on a near real-time basis [8].

The 1-3-7 malaria elimination strategy was initially developed and implemented in China in 2012 [9, 10], and Thailand both studied and adapted the strategy for the local epidemiological context. The strategy is considered a key factor in helping China reach zero locally transmitted malaria cases in 2017 and in supporting its efforts to maintain the interruption of transmission. Other countries in Southern Africa and Southeast Asia have also established malaria case reporting and response follow-up systems, complemented by increased monitoring activities [11, 12]. However, the hallmark of the 1-3-7 strategy is timeliness, with strict deadlines for reporting and response to enforce a robust elimination program. This paper outlines Thailand's experience adapting and implementing the 1-3-7 strategy, including lessons learned and success factors, in the hope that this information may be helpful for other countries considering strengthening surveillance for malaria elimination.

\section{From malaria control to malaria elimination}

From 2012 to 2015, the DVBD's malaria control efforts significantly reduced the blood slide positive rate (SPR) to less than $5 \%$ among suspected cases with fever and reduced annual parasite incidence (API) to less than 1 per 1,000 population (range $0.38-0.82$ ) [5]. The same downward trend was seen in malaria incidence, decreasing from 33,835 cases in 2012 to 24,332 cases in 2015, representing a 
$28.1 \%$ decline in just 3 years [8]. The malaria control program also successfully reduced mortality to just 0.05 deaths per 100,000 population by 2015, representing fewer than 50 malaria-attributed deaths from 2012 to 2015.

These results led to a comprehensive malaria program review in 2015 to identify strengths, gaps, and challenges across disciplines and to recommend key actions $[5,13]$. Recommendations for surveillance included updating and reorienting surveillance as a core intervention to detect and trigger an appropriate, focused, and timely response for every confirmed malaria case. Also in 2015, malaria became a notifiable disease in Thailand with the passage of the Infectious Disease Act, which expanded surveillance potential. To improve performance, the DDC decided to transition the malaria program to one focused on elimination (Figure 1) per the Global Technical Strategy for Malaria 2016-2030, with a goal to achieve national malaria elimination by 2024 [1]. The MOPH created a Malaria Elimination Strategic Plan for Thailand 2017-2026, and the DDC contributed the complementary Thailand Malaria Elimination Operational Plan 2017-2021 to pave the way forward [5]. These plans outlined incremental targets to support zero malaria by 2024.

* Source: Malaria Elimination Strategic Plan for Thailand 2017-2026 [5]. API, annual parasite incident; SPR, slide positive rate.

\section{Figure 1. Milestones from malaria control to malaria elimination}

The DVBD developed a new district-level stratification of malaria endemicity based on guidance from the WHO Malaria Elimination framework [14], which is detailed in the NMES [5]. The stratification is the basis for subsequent tailored case and vector management activities for elimination based on evidence from surveillance data. The DVBD also coordinated adoption of existing systems and infrastructure to prepare for adoption of the 1-3-7 strategy, including strengthening and sustaining epidemiological and entomological surveillance as recommended by the malaria program review committee.

\section{Protocols Of The 1-3-7 Strategy}

\section{Case reporting within 24 hours}

All individuals who test positive for malaria using microscopy or a rapid diagnosis test (RDT) receive malaria treatment according to the national treatment guidelines and trigger the start of the 1-3-7 surveillance process. All malaria species are included in 1-3-7 surveillance efforts, with Plasmodium falciparum and Plasmodium vivax being most common in Thailand [8]. Health care providers were already required to report confirmed cases and laboratory results under Thailand's malaria control strategy [8]; this was adjusted for the elimination program to include a requirement to complete the notification within 24 hours (1 day) of diagnosis [15]. An optional cellphone-based short message service alert system immediately informs district-level staff of a case, ensuring timely follow-up. Providers may also choose to report cases using the traditional paper form.

\section{Case investigation and classification within 3 days}

Thailand has long conducted malaria case investigations to identify risk behavior and to classify cases as imported or indigenous. Under the NMES, the MOPH adopted an enhanced case classification to allow a more precise understanding of where the patient contracted the disease and identify a source of transmission from remaining hotspots. Thailand's case investigation form (EP3) includes minimal essential data (e.g., demographics, illness history, diagnostic results, treatment, travel history) and likely location of infection. Cases are investigated by malaria clinic workers, district officers, or provincial officers and are classified per the criteria in Table $1[4]$.

Table 1. Case classification in Thailand 


\begin{tabular}{|c|c|}
\hline $\begin{array}{l}\text { Case } \\
\text { classification }\end{array}$ & Current definition \\
\hline A & Indigenous case, a patient who contracted malaria in the village where the patient lived during infection period \\
\hline B & $\begin{array}{l}\text { Imported case, a patient who contracted malaria from another area. Place of infection must be identified at village } \\
\text { level }\end{array}$ \\
\hline Bx & Contracted malaria from another village but within the same subdistrict \\
\hline By & Contracted malaria from another subdistrict but within the same district \\
\hline Bz & Contracted malaria from another district but within the same province \\
\hline Bo & Contracted malaria from another province \\
\hline Bf & Contracted malaria from abroad \\
\hline C & $\begin{array}{l}\text { Relapse case, a Plasmodium vivax or Plasmodium ovale patient who had a malaria episode and has recurrent } \\
\text { fever that is not a new infection }\end{array}$ \\
\hline D & Induced case, a patient who received malaria parasites from blood transfusion \\
\hline E & Introduced case, a patient who contracted malaria with evidence connecting the case to an imported case \\
\hline $\mathbf{F}$ & Unclassified case where, after investigation, staff are unable to determine a whether the patient is A or B \\
\hline G & Uninvestigated case, a patient that staff unsuccessfully attempted to investigate \\
\hline
\end{tabular}

Case investigation data are recorded on a paper form that is then uploaded to a national repository in the MIS. If the conclusive case classification is "A," a focus investigation is warranted, and the surveillance rapid response teams (SRRT) deploys the focus investigation and response.

\section{Foci investigation and response within 7 days}

Thailand's foci investigation compiles epidemiological, entomological, and sociological information and the results of past interventions. This allows local health authorities to identify causes and characteristics of indigenous transmission to deploy appropriate interventions. With the NMES, Thailand adopted a new foci stratification with four categories according to level of transmission, as outlined in Table 2 [16].

Table 2. Foci classification in Thailand

\begin{tabular}{|c|c|}
\hline $\begin{array}{l}\text { Foci } \\
\text { classification }\end{array}$ & Current definition \\
\hline A1 & Active foci: Village with indigenous cases in the current year \\
\hline A2 & $\begin{array}{l}\text { Residual non-active foci: Village without indigenous cases in the current year but with indigenous cases in the } \\
\text { previous } 3 \text { years }\end{array}$ \\
\hline B1 & $\begin{array}{l}\text { Cleared foci but receptive: Village without indigenous cases for } 3 \text { consecutive years but vectors are found or } \\
\text { environment is suitable for vector breeding }\end{array}$ \\
\hline B2 & $\begin{array}{l}\text { Cleared foci but not receptive: Village without indigenous cases for } 3 \text { consecutive but vectors are not found or } \\
\text { environment is not suitable for vector breeding }\end{array}$ \\
\hline
\end{tabular}

Thailand's adoption of the NMES also reoriented foci investigation in two ways. Firstly, a strict timeline to conduct the investigation within 7 days of case investigation was required. Secondly, in active foci (A1 and A2 areas) where all interventions are applied but malaria cases persist for over 4 consecutive weeks, reactive case detection (RACD) is conducted (Table 3 ). RACD is a surveillance response initiated when an indigenous index case is found in a village (foci) with transmission (A1 or A2) or without transmission but with the presence of a suitable vector (B1). Blood is taken for microscopy from all members living in the index patient's house and all 
neighbors living around the index patient's house, aiming for at least 50 blood samples or 10 households within a 1-kilometer radius. Additional foci response activities include vector control (distributing ITNs to reach $90 \%$ coverage at 1 bed net per 2 persons or indoor residual spraying [IRS] of at least $90 \%$ of households) and health education [4]. These data are stored in the MIS in a designated foci registry.

\section{Enhancing surveillance infrastructure for elimination}

Malaria Online was introduced in 2012 to facilitate case-based surveillance data tracking through online and offline reporting. The MIS comprises real-time malaria case data, geospatial foci and vector mapping, and drug efficacy data. It also serves as a resource portal for policies and strategic documents related to malaria. The MIS collates two main sources of malaria case recording and reporting (Figure 2):

1. The vertical malaria program, starting from subdistrict malaria clinics (MCs) through provincial Vector Borne Disease Centers (VBDCs). These health facilities record data on paper forms for case notification and case investigation; district health teams then input the data into the MIS.

2. The general health services (GHS) program, starting from community-level malaria posts (MPs) and hospitals (all types) through Provincial Health Offices (PHOs) to the Bureau of Epidemiology (BOE) [8]. These health facilities record data in a separate system maintained by the BOE.

Thailand is currently in a semi-vertical approach for malaria elimination as these two reporting lines are being integrated. Regardless of data type, 1-3-7 guidelines for timeliness of reporting are enforced.

In 2016, new features were added to enable real-time monitoring at every level of the health system and to encourage local staff to take ownership of their data and use the results to improve intervention targeting. The DVBD installed dashboards and visualizations in the MIS to show geospatial mapping of the 1-3-7 metrics, RACD targets and achievements, and a summary of the 1-3-7 malaria elimination strategy (Figure 3). A new 1-3-7 dataset, representing information from the weekly and monthly reports generated by provinces, includes the number of malaria cases reported and the proportion reported within 1 day, the number of cases investigated and the proportion investigated within 3 days, and the number of foci investigations (with RACD) and the proportion investigated within 7 days.

To encourage local staff to adopt 1-3-7 protocols and use the resulting data, the DVBD also created a weekly report that collates data highlights.

By allowing for timely analysis of malaria surveillance and case investigation data, Malaria Online supports Thailand's goal to use surveillance to develop strategic information that leads to appropriate action. A Business Intelligence add-on to the MIS facilitates comparisons of weekly incidence across years. If case numbers are higher than expected, the DVBD or provincial authorities alert the relevant subdistricts to consider appropriate investigation and control activities. In the example below, Prachuab Khiri Khan province received notice during the 27th week of the year that there were 20 more cases reported than in the same week the previous year (Figure 4). The notice included a dropdown list of villages; the action required to find out which areas were affected; and instructions for prompt case re-investigation, focus investigation with RACD of the whole village, and renewed vector control measures in line with the 1-3-7 strategy. Subnational officers took prompt action during the 27th week, with new cases triggering additional case investigations and a wider response until the next week witnessed a decreasing number of malaria cases.

For data to inform more granular operational decision-making as malaria burden decreases, data must be timely and accessible to subnational officers, health workers, and community focal persons. However, in the remaining hotspot locations in Thailand internet access may be unreliable and therefore prevents access to submitting and obtaining data in real time. To address these disadvantages, at the provincial and district levels the MIS can access and store back-up data in the Malaria Offline database in a central server [8]. Malaria Offline complements Malaria Online to ensure that work can continue during internet outages and that key staff can access their data.

\section{Human resources for strong surveillance}

Page 5/15 
Malaria surveillance for elimination in Thailand relies on human resources in both the malaria vertical program and the GHS system (Table 4). The surveillance system also engages village health volunteers (VHVs) for community reporting on 1-3-7 metrics. Some Vector Borne Disease Units (VBDUs) supervise GHS staff to ensure high-quality malaria case detection, surveillance, and response.

Day 1 malaria cases could be found and notified by staff from MCs, MPs, or border posts or by general health facility clinicians. Day 3 case investigations are conducted by MC staff or sometimes by public health workers at hospitals or MP staff with additional follow-up from SRRTs. Day 7 foci investigation and response largely relies on district-level SRRTs, a national system of epidemiology and investigation teams established in 2004 [18]. SRRT members include staff of both the vertical and GHS programs.

Both the DVBD and BOE remain committed to building skills and updating training materials and guidelines for effective implementation of the elimination program and the 1-3-7 strategy. The training package targeted epidemiologists, malaria surveillance focal points, and entomologists and was complemented by annual refresher training [13]. Public health workers trained all epidemiologists as part of the SRRT launch in each province to integrate malaria elimination activities into their work, including the 1-3-7 strategy and other public health responses. The training, comprising both workshops and on-the-job coaching, covered MIS data monitoring for timely action to correctly identify malaria infections and disrupt transmission. Since the launch of the 1-3-7 strategy, the DVBD has conducted annual trainings, resulting in 719 trained epidemiologists in Thailand. At the subdistrict and community levels, training for healthcare providers included VHVs. Entomologists were trained on vectors present in each subdistrict or VBDU area, a collection of mosquitoes for polymerase chain reaction (PCR) testing to rule out indigenous transmission in new B1 areas prior to their designation, and insecticide resistance monitoring for endemic areas.

\section{Allocating budget and resources}

Costs for surveillance and response activities, including required equipment, internet connectivity, and labor are supported through the MOPH budget, the health security budget, and local budgets. This domestic funding is enhanced by support from the Global Fund to Fight AIDS, Tuberculosis, and Malaria and additional external support from partners such as the United States President's Malaria Initiative. The MOPH also works with other agencies to coordinate malaria activities in their relevant domains and identify efficiencies, prioritize tailored activities, and maximize available funding. For example, shared information technology and systems resources across the government support malaria surveillance efforts.

From 2017 to 2020, the MOPH supported the malaria elimination strategy with Thai baht 2.8 billion (USD \$88.5 million), which does not include budget spent for health infrastructure and human resources [5]. The Malaria Elimination Steering Committee, comprising members from several ministries and WHO, develops an integrated budget. Of this budget, $11 \%$ is allocated to surveillance activities.

At the local level, PHOs and Local Administrative Organizations (LAOs) in areas with malaria transmission may allocate their own funds to malaria elimination projects. LAOs have subdistrict-level responsibility for public health and welfare issues, including malaria $[5,17]$. The 2015 Infectious Disease Act also stipulates that LAOs must conduct malaria surveillance and liaise with district health authorities. LAO committees that prioritize malaria as a community issue are empowered to design projects on disease control and response, based on hyperlocal malaria epidemiology and surveillance data. They also prepare and approve annual budget plans, and they manage funds for emergencies like disease outbreak response or natural disasters.

\section{Comparisons between the Thailand experience and the China experience}

The DVBD adopted the 1-3-7 strategy from China but tailored the strategy's activities to suit Thailand's requirements, available resources, and surveillance infrastructure. China included all suspected malaria cases in surveillance activities, which required substantial resources despite the country's low caseload at rollout in 2012 [19]. In Thailand, because all suspected malaria patients can access blood tests and results within the same day of visit, only confirmed positive cases trigger the 1-3-7 strategy. This not only promotes accurate management of fever patients and efficient use of commodities but also increases the quality of surveillance data by reducing reporting requirements.

Microscopy examination remains the most widely used diagnostic tool for laboratory confirmation of malaria both in Thailand and China. However, with declining incidence, practitioners' skills in performing and reading blood smears may decrease [9], so RDTs have recently become available in primary health care centers and communities in Thailand. This will allow VHVs to play a greater role in 
Thailand's malaria elimination strategy compared with China and also supports Thailand's system based on confirmed cases rather than suspected cases [20].

Foci classification also varied between the two countries, with four categories in Thailand and three categories in China. In China, areas with at least one confirmed malaria case are classified into three foci categories:

- "Inactive focus" is an area that is unlikely to support transmission because of an absence of vectors;

- "Active focus" is an area with suitable vectors; and

- "Pseudo focus" is a malaria-free area with a case classified as imported $[9,20]$.

Thailand has been using a foci classification system for several years, throughout both the malaria control and malaria elimination phases, based on malaria source (i.e., indigenous or imported), entomological factors, and environmental characteristics (i.e., unsuitable or suitable for transmission) [7]. Whereas in China both active and pseudo foci prompt RACD, in Thailand RACD screening is carried out only in foci with an index case (A1, A2, or B1).

Recording and reporting requirements were also slightly different because Thailand looked for efficiencies to maximize limited resources. In Thailand, to meet the criteria for foci reporting within 7 days, MIS users may input data through the end of the subsequent week that the case was reported. For example, if the case was identified on Monday of week 1, the VBDU can respond on any day starting on Monday of week 1 through Saturday (i.e., the last day) of week 2. As long as there were no other cases occurring in the same sub-village that week, the MIS will count that case as responded to within 7 days. Because workload for subnational officers can often be a bottleneck to timely surveillance and response, the DVBD applied a workload reducing strategy based on the number of cases in a province. For example, in a high-prevalence province like Tak, which reported 1,685 malaria cases in 2019, only one RACD was required even if more than one index case was reported at the same week in the same sub-village. Because each RACD targets 50 people per index case, coverage was adequate to maintain high-quality surveillance.

\section{Success factors to Thailand's 1-3-7 surveillance strategy}

The DVBD has heightened the visibility of malaria in Thailand by coordinating a Malaria Steering Committee and working across various government agencies including the Ministry of Interior, Ministry of Foreign Affairs, and Ministry of Defense. To engage policymakers, the team outlined a convincing argument that focused on the benefits of malaria elimination for the development of the country-for example, the DVBD published a cost-benefit analysis that found that every Thai baht invested in malaria elimination can return up to 15 Thai baht to Thailand's health system, households, and economy [21]. The DVBD also partnered with neighboring countries to share data and policies; this regional approach opened new funding opportunities through the Global Fund Regional Artemisinin-resistance Initiatives. More internally, the DVBD has prioritized malaria surveillance, including the 1-3-7 strategy, in its strategies and budgets, creating an enabling environment from Thailand's public health leadership. Supporting digital transformation policies have simultaneously catalyzed malaria surveillance infrastructure and success, including developing dashboards that facilitate review of 1-37 metrics and catalyze subsequent actions.

At the operational level, Thailand's malaria officers also have a mobile chat application that is a unique factor in the success of 1-3-7 surveillance. The chat group has over 400 malaria members from across the country and at every level of the health system. The group chat enables continual communication with peers to monitor the malaria situation, exchange experiences, seek recommendations or troubleshooting support, share press releases, and provide fodder for new strategies. Participation of the DVBD and national-level partners also supports more effective monitoring and evaluation. The active members respond to notifications, ask questions and provide answers, and offer motivation and support, making it an efficient platform for monitoring and communication among malaria workers.

A final success factor in Thailand's implementation is the flexibility of implementing budgets and activities at very local levels. Even though annual budgets are calculated based on the previous year's activities and unexpected situations often occurred, LAOs were able to work with provincial authorities to revise their local budgets to fit needed activities for the current situation. Because these local authorities know the community situation, this microplanning has been a successful strategy for organizing targeted response even in situations with unanticipated spikes in malaria caseload.

\section{The future road to elimination}


The 1-3-7 strategy has been successfully implemented, and results suggest it has contributed to Thailand's decline in malaria burden from 14,948 cases in fiscal year 2017 to just 4,421 in fiscal year 2019, representing a 70\% reduction in incidence. The DVBD continues to adapt its program to ensure prompt and high-quality case management, capacity maintenance, and adequate supply of lifesaving commodities. It is also continuing routine data monitoring and wider assessments as needed to verify that the time-bound targets of the 1-3-7 strategy are sustained over time. Senior staff are expanding technical discussions and resources to include prevention of reintroduction. However, the DVBD may also need to consider potential challenges that may emerge as provinces approach very low prevalence.

The MOPH is working toward an integrated system of health services by phasing out vertical VBDUs, VBDCs, and MCs. As staff in these facilities retire, the MOPH is not recruiting replacements so that future patients are directed toward GHS facilities, including health promoting hospitals [13]. The new structure of GHS's malaria program is semi-vertical, with the DVBD's expertise guiding the development of high-quality malaria services across facility types. This guidance includes incorporation of malaria case management into GHS pre-service training, mechanisms of in-service training and professional support through VBDU, and an encouraging career path for GHS trainees $[5,8]$. The DVBD will remain involved in the integration process to ensure the high quality of malaria case management and surveillance is maintained through elimination and prevention of reintroduction.

When areas reach very low prevalence, the DVBD may consider supporting provincial authorities to take a stronger role in quality assurance. Additional online training and standard operating procedures could support a more decentralized system to fortify comprehension of protocols despite current challenges caused by the coronavirus disease. An annual online training with certification could be one way to maintain knowledge and skills, share updates, and review surveillance data as fewer health staff interact with suspect malaria cases and have robust malaria case management experience.

As the DVBD's efforts reduce malaria burden, advocating for necessary funds becomes more challenging. Thailand's budget for malaria surveillance has changed over time. However, the technical malaria community knows that malaria elimination programs are more resource-intensive than malaria control, with increasing cost per case. The current global funding landscape is shifting, and external funds available to Thailand are shrinking. This challenge requires the DVBD to be more creative and strategic with identifying sources of financial support. Especially in the described climate of integration, domestic fund-raising is also a challenge because it requires that malaria be prioritized over all other diseases Thailand is working to address.

Table 3. Implementation of the 1-3-7 strategy for malaria elimination 


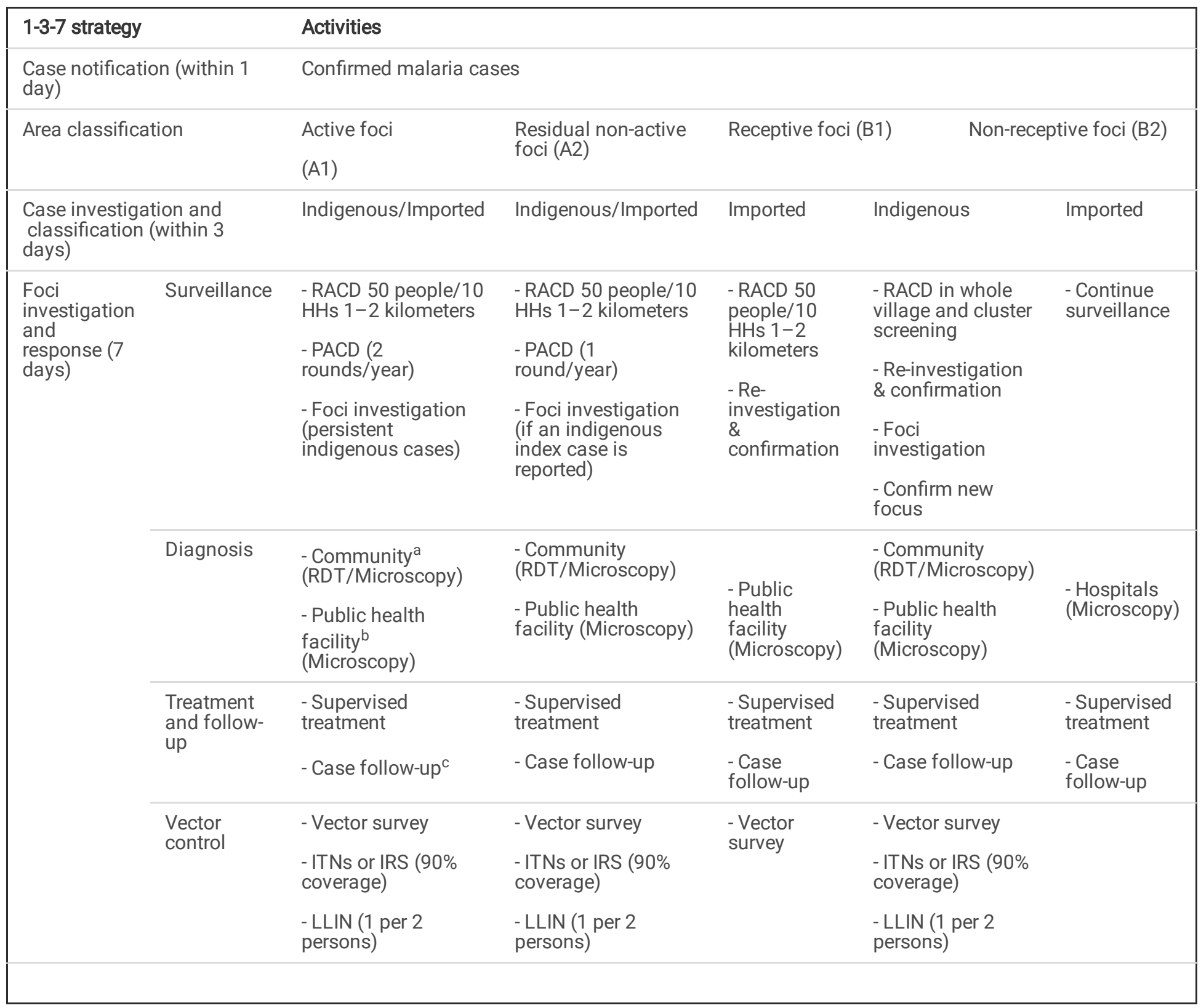

HH, household; IRS, indoor residual spraying; ITN, insecticide-treated net; LLIN, long-lasting insecticide-treated bed net (includes ITNs, retreated bed nets, and hammocks); PACD, proactive case detection; RACD, reactive case detection; RDT, rapid diagnosis test.

${ }^{a}$ Malaria posts and malaria clinics; ${ }^{b}$ Health promoting hospitals and district, provincial, and regional public hospitals; ${ }^{c}$ Follow-up and re-testing for $P$. falciparum and $P$. vivax cases according to national guidelines.

Table 4. Roles and responsibilities in implementing the 1-3-7 strategy 


\begin{tabular}{|c|c|c|}
\hline Country level & Ministry of Public Health & \\
\hline \multirow[t]{9}{*}{ Central level } & Division of Vector Borne Diseases, & \multirow[t]{2}{*}{ Department of Epidemiology } \\
\hline & Department of Disease Control & \\
\hline & - Make policy decisions & \multirow{7}{*}{$\begin{array}{l}\text { - Make policy decisions } \\
\text { - Cross-check and analyze data } \\
\text { - Share feedback with and support subnational staff } \\
\text { - Collaborate with Department of Disease Control as C- } \\
\text { SRRT }\end{array}$} \\
\hline & - Supervise, monitor, and evaluate interventions & \\
\hline & $\begin{array}{l}\text { - Integrate data from all sources to the MIS } \\
\text { database }\end{array}$ & \\
\hline & - Cross-check and analyze data; share feedback & \\
\hline & $\begin{array}{l}\text { - Share feedback with and support subnational } \\
\text { staff }\end{array}$ & \\
\hline & $\begin{array}{l}\text { - Provide technical support for the MIS and Malaria } \\
\text { Offline }\end{array}$ & \\
\hline & $\begin{array}{l}\text { - Collaborate with Department of Epidemiology as } \\
\text { C-SRRT }\end{array}$ & \\
\hline \multirow{5}{*}{$\begin{array}{l}\text { Provincial } \\
\text { level }\end{array}$} & Vector Borne Disease Centers & Provincial Health Offices \\
\hline & $\begin{array}{l}\text { - Enter and upload data (Days } 1,3,7 \text { ) into Malaria } \\
\text { Offline program }\end{array}$ & \multirow{2}{*}{$\begin{array}{l}\text { - Supervise, monitor, and evaluate interventions } \\
\text { - Monitor provincial data and facilitate reporting and } \\
\text { feedback }\end{array}$} \\
\hline & - Supervise, monitor, and evaluate interventions & \\
\hline & $\begin{array}{l}\text { - Monitor provincial MIS data and facilitate } \\
\text { reporting and feedback }\end{array}$ & \multirow[t]{2}{*}{ - Collaborate with Vector Borne Disease Center as P-SRRT } \\
\hline & $\begin{array}{l}\text { - Collaborate with Provincial Health Office as P- } \\
\text { SRRT }\end{array}$ & \\
\hline \multirow[t]{9}{*}{ District level } & Vector Borne Disease Units & District Health Offices \\
\hline & $\begin{array}{l}\text { - Enter and upload data (Days } 1,3,7 \text { ) into Malaria } \\
\text { Offline program }\end{array}$ & \multirow{3}{*}{$\begin{array}{l}\text { - Enter data into HosXP reporting program } \\
\text { - Supervise, monitor, and evaluate interventions } \\
\text { - Monitor district data and facilitate reporting and feedback } \\
\text { - Collaborate with Vector Borne Disease Units as D-SRRT }\end{array}$} \\
\hline & - Supervise, monitor, and evaluate interventions & \\
\hline & $\begin{array}{l}\text { - Monitor district MIS data and facilitate reporting } \\
\text { and feedback }\end{array}$ & \\
\hline & - Collaborate with District Health Office as D-SRRT & \multirow{3}{*}{$\begin{array}{l}\text { District Hospitals } \\
\text { - Conduct malaria testing, treatment, and follow-up } \\
\text { - Day 1: Send malaria reports (R506) to District Health } \\
\text { Office }\end{array}$} \\
\hline & $\begin{array}{l}\text { - Day 1: Send malaria case reports (EP1) to } \\
\text { provincial and central levels }\end{array}$ & \\
\hline & - Day 3: Complete case investigation and & \\
\hline & & $\begin{array}{l}\text { - Day 3: Complete case investigation and classification; } \\
\text { send form to Department of Epidemiology }\end{array}$ \\
\hline & & - Collaborate with Vector Borne Disease Units as D-SRRT \\
\hline \multirow{6}{*}{$\begin{array}{l}\text { Subdistrict } \\
\text { level }\end{array}$} & Malaria Clinics & Health Promoting Hospital, Health Centers \\
\hline & $\begin{array}{l}\text { - Conduct malaria screening, testing, treatment, and } \\
\text { follow-up }\end{array}$ & \multirow{3}{*}{$\begin{array}{l}\text { - Supervise, monitor, and evaluate interventions } \\
\text { - Conduct malaria testing, treatment, and follow-up } \\
\text { - Day 1: Send malaria reports (R506) to district level }\end{array}$} \\
\hline & & \\
\hline & level & \\
\hline & $\begin{array}{l}\text { - Day 3: Complete case investigation and } \\
\text { classification; send EP3 form to district level }\end{array}$ & $\begin{array}{l}\text { - Day 3: Complete case investigation and classification; } \\
\text { send form to Department of Epidemiology }\end{array}$ \\
\hline & $\begin{array}{l}\text { - Day 7: Conduct foci investigation and RACD as L- } \\
\text { SRRT }\end{array}$ & - Day 7: Conduct foci investigation and RACD as L-SRRT \\
\hline \multirow{2}{*}{$\begin{array}{l}\text { Community or } \\
\text { village level }\end{array}$} & Malaria Posts and Border Malaria Posts & Village Health Volunteers \\
\hline & - Conduct malaria screening, testing, and follow-up & - Conduct malaria screening, testing, and follow-up \\
\hline
\end{tabular}


- Day 1: Send malaria case report (EP1) to district level

- Day 7: Support L-SRRT
- Day 1: Send malaria case report (EP1) to district level

- Day 7: Support L-SRRT

EP1, Malaria Blood Examination form; EP3, Investigation and Radical Treatment of Malaria case form; R506, Disease Surveillance Report form; D-SRRT, District-SRRT; L-SRRT: Local-SRRT; MIS, malaria information system; SRRT, Surveillance Rapid Response Team; PSRRT, Provincial-SRRT.

\section{Conclusions}

In conclusion, the DVBD has successfully tailored the 1-3-7 strategy for malaria surveillance and response to the Thai context with a core objective of accelerating malaria elimination. The time-bound components of this strategy have emphasized the target of zero malaria by 2024 , and results from the first few years of implementation show Thailand is on track to reach its goal. The DVBD will continue to track progress and ensure a high quality of care for malaria patients as the program integrates malaria services into the GHS. This experience may be useful for other countries aiming to eliminate malaria in the region.

\section{Abbreviations}

API Annual parasite incidence

BOE Bureau of Epidemiology

DDC Department of Disease Control

DVBD Division of Vector Borne Diseases

GHS General Health Services program

GMS Greater Mekong Subregion

GTS Global Technical Strategy for Malaria 2016-2030

HH Household

IRS Indoor Residual Spraying

ITN Insecticide-Treated Net

LAO Local Administrative Organization

MC Malaria Clinic

MIS Malaria Information System

MOPH Ministry of Public Health

MP Malaria Post

NMES National Malaria Elimination Strategy

PACD Passive Case Detection

PCR Polymerase Chain Reaction

PHO Provincial Health Office

RACD Reactive Case Detection

RDT Rapid Diagnostic Test 
SPR Slide Positive Rate

SRRT Surveillance and Rapid Response Team

USAID United States Agency for International Development

USD U.S. Dollars

VBDC Vector Borne Disease Center

VBDU Vector Borne Disease Unit

VHV Village Health Volunteer

WHO World Health Organization

\section{Declarations}

\section{Ethics approval and consent to participate}

Not applicable.

\section{Consent for publication}

Not applicable.

\section{Availability of data and materials}

The visualizations supporting the conclusions of this article are available in the Malaria Online repository, http://malaria.ddc.moph.go.th/.

\section{Competing interests}

The authors declare that they have no competing interests.

\section{Funding}

This study was made possible by the generous support of the American people through the U.S. President's Malaria Initiative and United States Agency for International Development (USAID), under the terms of Cooperative Agreement AID-486-LA-15-00002 for Inform Asia: USAID's Health Research Program. The contents of this article are the responsibility of the authors and do not necessarily reflect the views of USAID or the U.S. Government.

\section{Authors' contributions}

PS, SK, and DG conceptualized the manuscript. SN and JAS led the document review and writing of the manuscript. All other authors provided input and review.

\section{Acknowledgements}

The authors recognize all local public health teams and partners who have contributed to planning and implementing the 1-3-7 strategy at the national and field levels under the leadership of Thailand's Division of Vector Borne Diseases, Department of Disease Control, Ministry of Public Health.

\section{References}

1. World Health Organization (WHO). Strategy for Malaria Elimination in the Greater Mekong Subregion (2015-2030). Geneva, Switzerland: WHO; 2015. (WC 765.)

2. World Health Organization (WHO). World malaria report. Geneva, Switzerland: WHO; 2020.

3. Kaehler N, Adhikari B, Cheah PY, von Seidlein L, Day NPJ, Paris DH, Tanner M, Pell C. Prospects and strategies for malaria elimination in the Greater Mekong Sub-region: a qualitative study. Malar J. 2019;18:203; doi:10.1186/s12936-019-2835-6. 
4. Sudathip P, Kitchakarn S, Thimasarn K, Gopinath D, Naing T, Sajjad O, Hengprasert S. The evolution of the malaria clinic: The cornerstone of malaria elimination in Thailand. Trop Med Infect Dis. 2019;4:1-22; doi:10.3390/tropicalmed4040143.

5. Bureau of Vector Borne Disease. National Malaria Elimination Strategy, Thailand 2017-2026. Ministry of Public Health; 2016.

6. Khamsiriwatchara A, Sudathip P, Sawang S, Vijakadge S, Potithavoranan T, Sangvichean A, Satimai W, Delacollette C, Singhasivanon P, Lawpoolsri S, Kaewkungwal J. Artemisinin resistance containment project in Thailand. (I): Implementation of electronic-based malaria information system for early case detection and individual case management in provinces along the ThaiCambodian border. Malar J. 2012;11:247; doi:10.1186/1475-2875-11-247.

7. Bureau of Vector Borne Disease. Guideline of Malaria control practice for public health worker. MOPH: Division of Malaria Disease; 2009.

8. Division of Vector Borne Disease. Malaria Online: The Digital Surveillance System for Thailand Malaria Elimination. MOPH; 2019.

9. Lu G, Liu Y, Beiersmann C, Feng Y, Cao J, Muller O. Challenges in and lessons learned during the implementation of the 1-3-7 malaria surveillance and response strategy in China: a qualitative study. Infect Dis Poverty. 2016;5:94; doi:10.1186/s40249-016-0188-8.

10. Feng J, Liu J, Feng X, Zhang L, Xiao H, Xia Z. Towards malaria elimination: Monitoring and evaluation of the "1-3-7" approach at the China-Myanmar border. Am J Trop Med Hyg. 2016;95:806-810; doi:10.4269/ajtmh.15-0888.

11. van Eijk AM, Ramanathapuram L, Sutton PL, Kanagaraj D, Sri Lakshmi Priya G, Ravishankaran S, Asokan A, Tandel N, Patel A, Desai $\mathrm{N}$, et al. What is the value of reactive case detection in malaria control? A case-study in India and a systematic review. Malar J. 2016;15:67; doi:10.1186/s12936-016-1120-1.

12. Wang D, Chaki P, Mlacha Y, Gavana T, Michael MG, Khatibu R, Feng J, Zhou ZB, Lin KM, Xia S, et al. Application of community-based and integrated strategy to reduce malaria disease burden in southern Tanzania: the study protocol of China-UK-Tanzania pilot project on malaria control. Infect Dis Poverty. 2019;8:4; doi:10.1186/s40249-018-0507-3.

13. World Health Organization (WHO). Programmatic Review of the National Malaria Programme in Thailand: 31 st August - 11th September 2015, Summary Report. 978-92-9022-513-3. Nonthaburi, Thailand: WHO; 2016. (WC 765.)

14. Global Maria Programme. A framework for malaria elimination. Geneva, Switzerland: WHO; 2017.

15. Government of Thailand. Communicable Disease Act. B.E. 2015: The Office of the Council of State; 2015.

16. Sudathip P, Kitchakarn S, Shah JA, Bisanzio D, Young F. A Foci cohort analysis to monitor successful and persistent foci under Thailand's malaria elimination strategy. Malar J, in press.

17. Bureau of Vector Borne Disease. Guide to malaria elimination for Thailand's Local Administrative Organizations and the health network. Ministry of Public Health; 2019.

18. Ungchusak K, Yingyong T, Ardkean W, Saritapirak N, Narueponjirakul U, Areechokchai D, Hanchaoworakul W, lamsirithavorn S, Prempree P, Suwannathapa S. Chapter 9: Kingdom of Thailand: Implementation of Thailand's SRRT (Surveillance and Rapid Response Team) for Outbreak Containment. ASEAN Plus Three; n.d.

19. Zhou SS, Zhang SS, Zhang L, Rietveld AE, Ramsay AR, Zachariah R, Bissell K, Van den Bergh R, Xia ZG, Zhou XN, Cibulskis RE. China's 1-3-7 surveillance and response strategy for malaria elimination: Is case reporting, investigation and foci response happening according to plan? Infect Dis Poverty. 2015;4:55; doi:10.1186/s40249-015-0089-2.

20. Cao J, Sturrock HJ, Cotter C, Zhou S, Zhou H, Liu Y, Tang L, Gosling RD, Feachem RG, Gao Q. Communicating and monitoring surveillance and response activities for malaria elimination: China's "1-3-7" strategy. PLoS Med. 2014;11:e1001642; doi:10.1371/journal.pmed.1001642.

21. Sudathip P, Kongkasuriyachai D, Stelmach R, Bisanzio D, Sine J, Sawang S, Kitchakarn S, Sintasath D, Reithinger R. The investment case for malaria elimination in Thailand: A cost-benefit analysis. Am J Trop Med Hyg. 2019;100:1445-1453; doi:10.4269/ajtmh.180897.

\section{Figures}




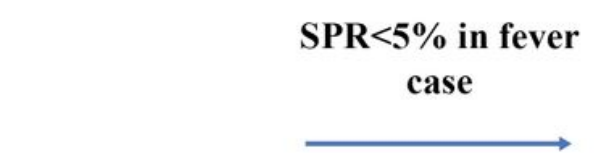

API $<1 / 1000$

population
0 locally acquired case $\rightarrow 3$ years
WHO certification

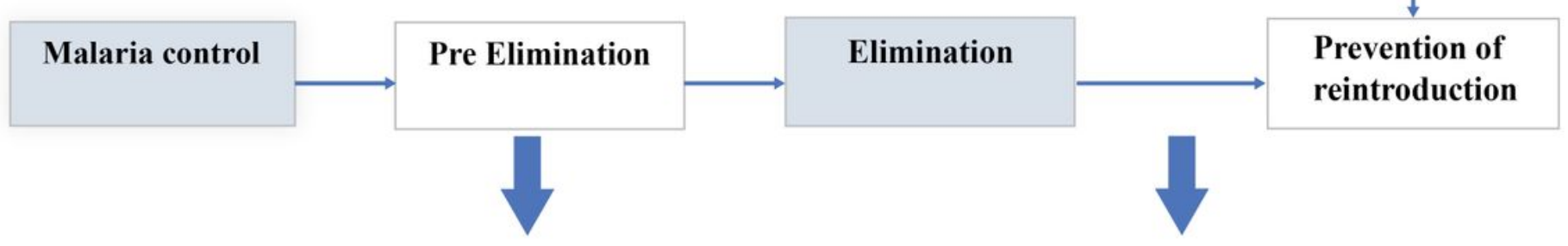

Prepare malaria elimination phase 1

Figure 1

Milestones from malaria control to malaria elimination * Source: Malaria Elimination Strategic Plan for Thailand 2017-2026 [5]. API, annual parasite incident; SPR, slide positive rate.

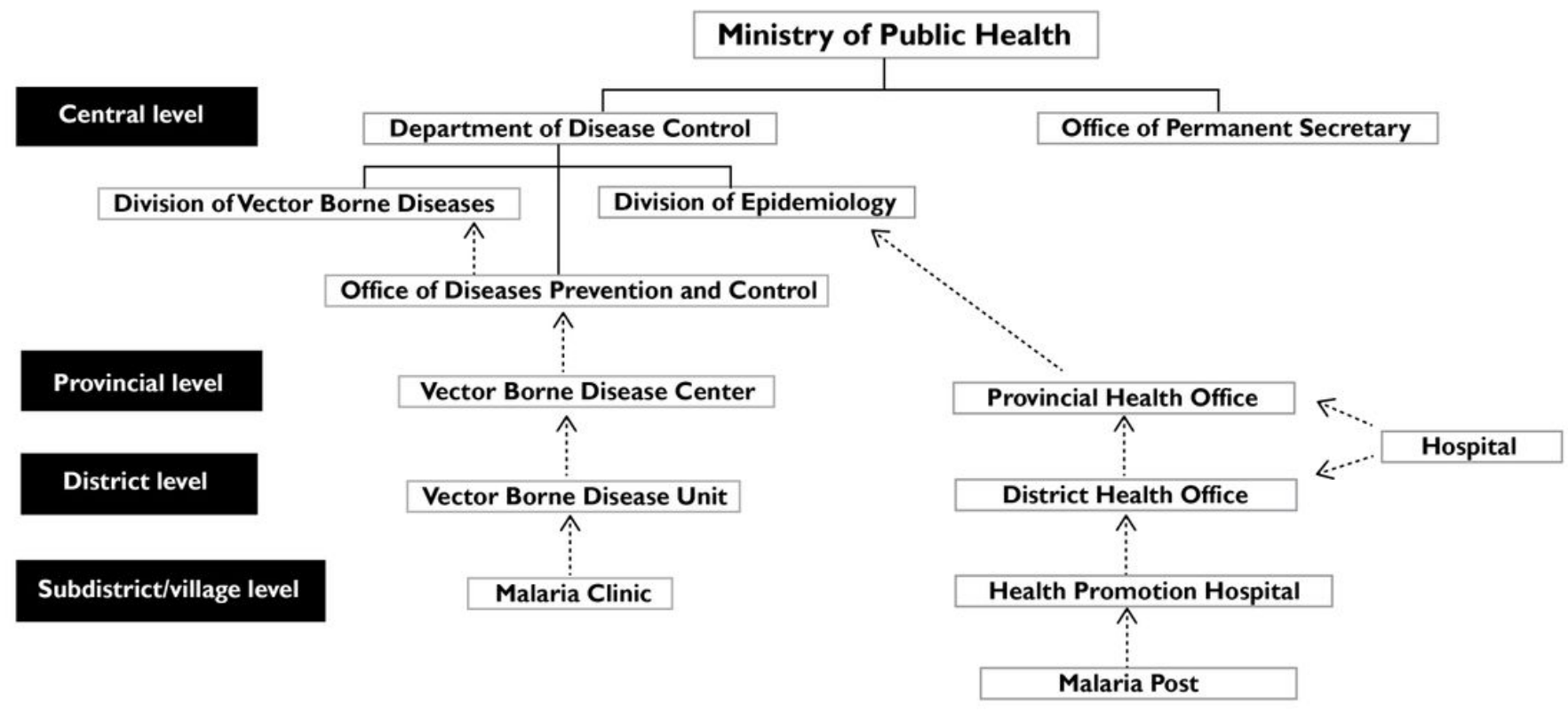

Figure 2 


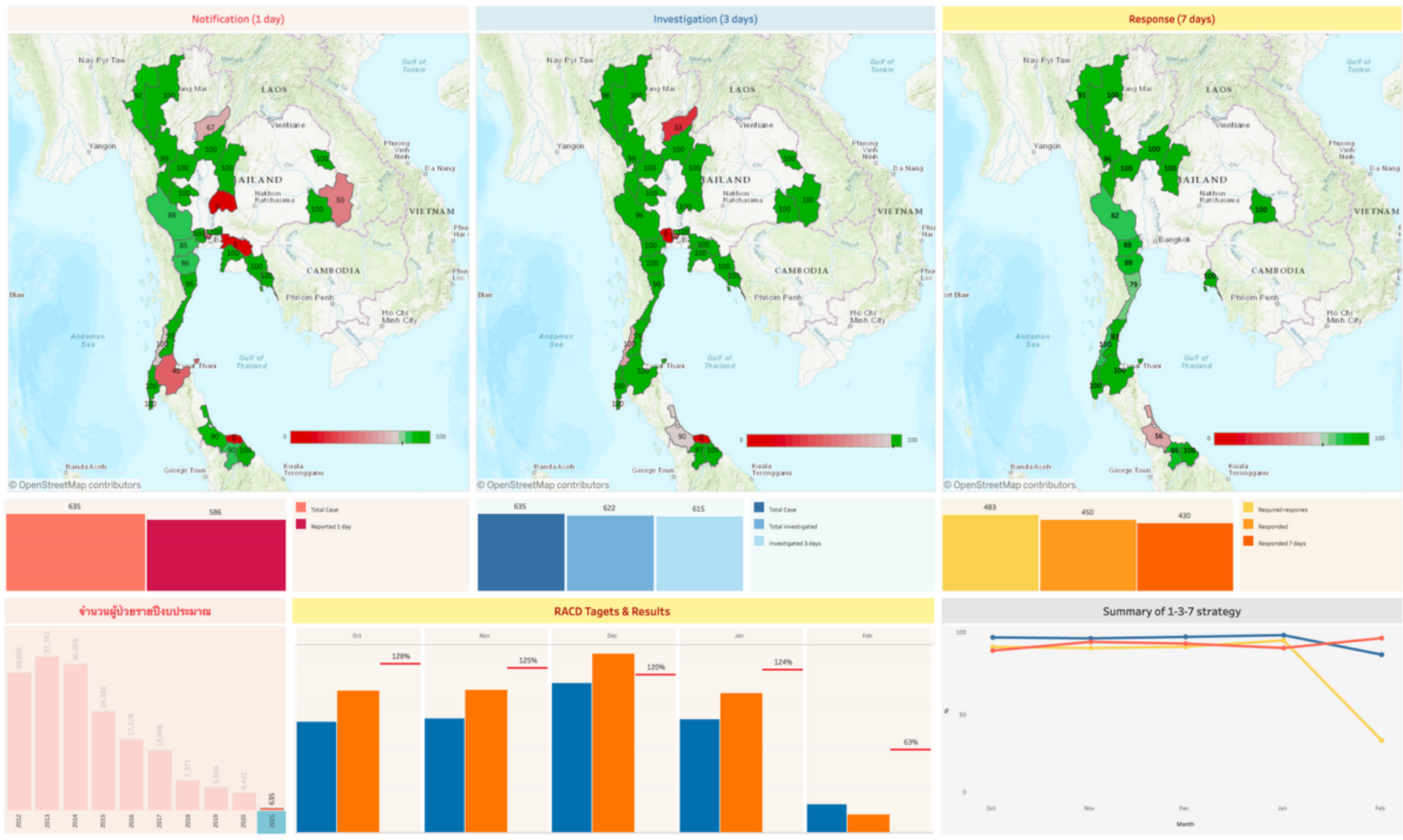

\section{Figure 3}

1-3-7 dashboard in the MIS *Source: MIS database [8], DVBD, MOPH. Note: The designations employed and the presentation of the material on this map do not imply the expression of any opinion whatsoever on the part of Research Square concerning the legal status of any country, territory, city or area or of its authorities, or concerning the delimitation of its frontiers or boundaries. This map has been provided by the authors.

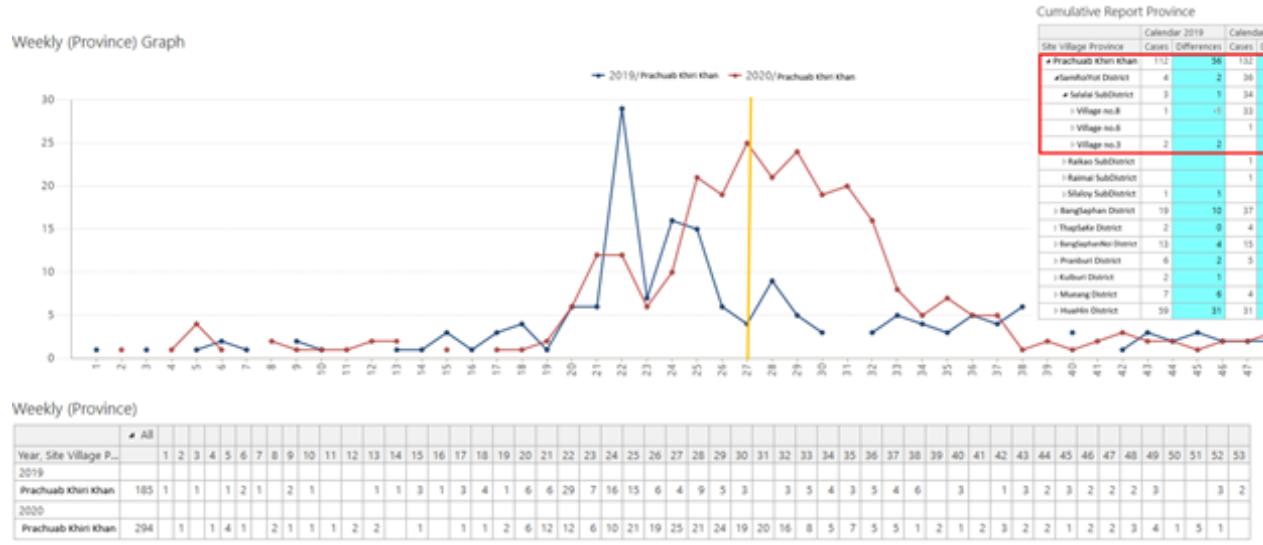

\section{Figure 4}

Weekly data report for Prachuab Khiri Khan in the MIS * Source: DVBD, MOPH 